Portland State University

PDXScholar

\title{
A Literature Review Evaluating the Efficacy of Meditation as an Adjunctive Method for Managing Chronic Lower Back Pain
}

Alison M. Wyckoff

Portland State University

Follow this and additional works at: https://pdxscholar.library.pdx.edu/honorstheses

Let us know how access to this document benefits you.

\section{Recommended Citation}

Wyckoff, Alison M., "A Literature Review Evaluating the Efficacy of Meditation as an Adjunctive Method for Managing Chronic Lower Back Pain" (2019). University Honors Theses. Paper 773.

https://doi.org/10.15760/honors.791

This Thesis is brought to you for free and open access. It has been accepted for inclusion in University Honors Theses by an authorized administrator of PDXScholar. Please contact us if we can make this document more accessible: pdxscholar@pdx.edu. 


\title{
A Literature Review Evaluating the Efficacy of Meditation as an Adjunctive Method for Managing Chronic Lower Back Pain
}

\author{
By \\ Alison Wyckoff \\ An undergraduate honors thesis submitted in partial fulfillment of the requirements for the \\ degree of \\ Health Studies: Health Sciences \\ in University Honors and \\ OHSU-PSU School of Public Health
}

Thesis Adviser

Claire Wheeler MD, Ph.D.

Portland State University

2019 


\section{Table of Contents}

$\begin{array}{ll}\text { Abstract } & 3\end{array}$

$\begin{array}{lr}\text { Introduction } & \mathbf{3}\end{array}$

$\begin{array}{ll}\text { Chronic Pain } & 3\end{array}$

Cost of Chronic Pain $\quad 3$

Current Methods of Treatment 4

Meditation $\quad 5$

$\begin{array}{lc}\text { Methods } & 6\end{array}$

Results

Morone et al. (2016) $\quad 6$

Luiggi-Hernandez et al. (2017) $\quad 7$

$\begin{array}{ll}\text { Cherkin et al. (2016) } & 7\end{array}$

Zgierska et al. (2016) $\quad 8$

Kratz, Murphy, Kalpakjian, and Chen (2018) 9

Day et al. (2019) 9

Michalsen et al. (2016) 9

Ardito et al. (2017) 9

$\begin{array}{ll}\text { Schmidt et al. (2015) } & 10\end{array}$

$\begin{array}{ll}\text { Discussion } & 10\end{array}$

$\begin{array}{ll}\text { Conclusion } & 12\end{array}$

$\begin{array}{ll}\text { References } & 14\end{array}$ 


\begin{abstract}
This thesis is a comprehensive review of the literature on the efficacy of meditation an adjunctive treatment for chronic lower back pain management. Nine published studies were identified by a comprehensive search of peer-reviewed literature. The studies vary methodologically in their organization and designs as well as by sample size, with the largest study including 282 participants and the smallest including 8 participants. The studies were compared and contrasted based on experimental design, measured outcomes or assessments, and research findings. The main outcomes measured across the studies included changes in functional mobility, pain bothersomeness, acceptance and awareness, and changes in depressive states, the results of which were variable. The differences in results may be contributable to inconsistencies between the organization and design of each study. Overall, the studies supported meditation, specifically the MBSR program, as a beneficial additional method of treatment for chronic lower back pain management. Given the increasing need for effective treatments that target chronic pain management, greater adherence to the consistency in the organization and design of randomized control studies to that of the MBSR clinical procedures, must be emphasized in order to evaluate the efficacy of the MBSR program as a valuable adjunctive treatment.
\end{abstract}

Keywords: Chronic pain; Chronic lower back pain; Meditation; Mindfulness Based Stress Reduction (MBSR); Mindfulness

\title{
Introduction
}

\section{Chronic Pain}

Pain is defined as a signal in the nervous system indicating that something may be wrong with the physical body (NIH, 2019). Pain may be experienced as a prick, tingle, sting, burn or ache within the body and each may be experienced to varying degrees, ranging from dull to sharp (NIH, 2019). The NIH has divided pain into two categories: acute pain and chronic pain. Acute pain is experienced with injury and is typically sharp or severe (NIH, 2019). The NIH (2019) defines chronic pain as pain that lasts for weeks, months, or even years. The severity of chronic pain can be further distinguished by the degree to which the pain limits major life domains, such as work, social and recreational activities, as described by Dahlhamer et al. (2016) in the CDC Morbidity and Mortality Weekly Report. People living with chronic pain may be able to maintain normal life activities despite the condition, but others, said to have high-impact chronic pain, cannot maintain daily life activities. According to the NIH (2019), chronic pain may result from injury, or infection, and in cases involving arthritis or cancer the cause may be ongoing, or in other instances the cause may be idiopathic. Notably, environmental and psychological factors can worsen the experience of pain (NIH, 2019). According to Clark, Yang and Janal (1998), stressful experiences in particular affect pain thresholds, as they may induce either analgesia or hyperalgesia.

\section{Cost of Chronic Pain}

The CDC analyzed the 2016 National Health Interview Survey (NHIS) data to estimate the prevalence of chronic pain in the U.S. and reported that $20.4 \%$ or 50.5 million U.S. adults live with chronic pain, and an additional $8 \%$ or 19.6 million U.S. adults were found to be living with highimpact chronic pain (Dahlhamer et al., 2018). According to Dahlhamer et al., women, veterans, adults of advanced ages, adults who were unemployed but had previously been employed, adults with educational background below that of a bachelor's degree, adults living in poverty, and adults living in rural communities were reported with higher prevalence of chronic pain. Data from the Centers for Disease Control (CDC) (2006) revealed that musculoskeletal conditions including 
spinal disorders, arthropathies, and rheumatisms were within the top 10 most common reasons adults sought medical care services. In 2001 to 2002, 29.4 million ambulatory visits to physicians' offices were the result of arthropathic conditions which were diagnosed upon arrival (Schappert, 2006). These statistics support the claim that chronic pain conditions are a leading cause for hospital visits (Schappert, 2006).

A 2011 report from the Institute of Medicine (IOM) made a conservative estimate that the economic cost of chronic pain in the U.S. was $\$ 560-635$ billion a year. The estimate takes into account the cost of healthcare and the cost of lost productivity, which were estimated at \$261-300 billion and \$297-336 billion respectively (IOM, 2011). According to the IOM, in 2008 the Federal Medicare program covered $25 \%$ of the U.S. medical expenditures on pain, which was valued at $\$ 65.3$ billion. State and federal programs, including Medicare, Medicaid, and the Department of Veterans Affairs, Workers' compensation and other medical aid programs, covered $\$ 99$ billion attributed to chronic pain in 2008 (IOM, 2011). These estimates do not include the lost revenue from taxable incomes, which would likely have compounding effects (IOM, 2011).

\section{Current Methods of Treatment}

It has been found that a common treatment for high impact chronic pain resulting from osteoarthritis is total joint replacement surgery (Kremers et al., 2015). Kremers et al. estimated that in the year of 2010, 2.5 million individuals in the U.S. had total hip replacement surgery and 4.7 million had total knee replacement surgery. With improved quality in function and mobility gained from joint replacement surgeries, and the lack of prevention of osteoarthritis, there will likely be an increase in the demand for total joint replacement surgeries (Kremers et al., 2015). From analyzing large cohorts from national data, Kremers et al. estimated that in the year of 2030, 11 million individuals may be living with total knee or hip replacements, and with the rise in prevalence, the study also predicted a shift to younger ages. It is also worth reporting that in 20052006 African Americans received total hip replacement surgeries at a rate of 5.6 per 1,000 people in the U.S., while Caucasian Americans received the same surgeries at a rate of 9.1 per 1,000 (Tomek, Goodman, Esty, Bell, \& Fisher, 2010).

One non-invasive method used to combat chronic pain in the past has been prescription of opioid drugs such as oxycodone, hydrocodone, codeine, morphine, and many others (Volkow \& McLellan, 2016). Opioids are a class of narcotic drugs with chemical structures that mimic natural neurotransmitters, allowing them to attach to opioid receptors in the brain. The stimulation of these nerve cells produces what is called the "opioid effect," which blocks pain and stimulates the production of dopamine (Volkow \& McLellan, 2016). According to the National Alliance of Advocates for Buprenorphine (2008), overstimulation of this system produces euphoric effects, which is the basis for the addictive qualities of these drugs. Opioids are also the most commonly prescribed and effective analgesic treatment for severe, postoperative pain (Corder et al., 2018).

Heavy reliance and over prescribing of opioid drugs have contributed to an epidemic of opioid related overdoses and addiction (Compton, \& Volkow, 2006). According to the CDC (2018), 17.4\% of Americans received one or more opioid prescriptions, with an average receiving 3.4 prescriptions in 2017. More than 3.5\% of Americans reported misusing prescription opioid drugs in 2016 (Hoots et al., 2018). In their 2018 publication, the CDC also reported a record high for drug overdose fatalities in 2016, with a total of 63,632 fatal drug overdoses, which is a rate of 19.8 per 100,000 persons (Hoots et al., 2018). Of the total reported, 66.4\%, or 42,249, of the drug overdose fatalities involved prescription or illicit opioids, with the most common being manufactured 
fentanyl (Hoots et al., 2018). Rates of drug overdoses involving synthetic opioids such as fentanyl have increased by $87 \%$ from 2013 to 2016. A multifaceted preventative approach is needed to respond to the epidemic of opioid abuse through harm reduction and recovery informed care, along with safe prescribing and pain management practices (Seth, Scholl, Rudd \& Bacon, 2018).

Aside from the serious risks that addiction to opioid drugs pose, there are two side effects that reduce the efficacy of long-term treatment with prescription opioids for chronic pain (Ballantyne \& Mao, 2003). Ballantyne and Mao have described one side effect to be the pharmacologic phenomenon of opioid tolerance, which develops from repeated use and establishes a need for increased dose in order to maintain equipotent analgesic, or pain reducing effects. According to the authors, opioid tolerance can be both associative, which involves psychological factors, as well as non-associative, which occurs at the neurological level. Non-associative opioid tolerance involves the down regulation or desensitization of opioid receptors through a decreased turnover rate, as well as a reduction in the number of receptors (Ballantyne \& Mao, 2003). The use of opioid drugs is also associated with the activation of a "pronociceptive mechanism leading to increased pain sensitivity," also known as hyperalgesia (Wilder-Smith \& Arendt-Nielsen, 2006, p. 602). The onset of opioid induced hyperalgesia is associated with long term use, high doses, and abrupt changes in dose concentration (Wilder-Smith \& Arendt-Nielsen, 2006).

\section{Meditation}

Meditative practices commonly used for therapeutic purposes originate from Zen Buddhism, yoga and Vipassana (Kabat-Zinn, 1985). Applied without the religious and culturally specific ideologies, such highly developed practices utilize one's attention to develop what is referred to as "insight," "bare attention," or "detached observation" (Kabat-Zinn, 1985). Meditation involves different mental skills and qualities of consciousness depending on the ideology (Kabat-Zinn, 1985). As described by Kabat-Zinn (1985), mindful meditation draws from the traditional practices of Theravada Buddhism, and is known as Satipatthana Vipassana, which works to develop awareness and attentiveness to ordinary experiences (Young, 1994). One of the goals of mindful meditation is to remain aware of a phenomenon, such as physical pain, from moment-to-moment, as it exists, without allowing one's judgments or feelings to distort it (KabatZinn, 1985).

The Mindfulness Based Stress Reduction (MBSR) program, developed by Jon Kabat-Zinn, combines a range of techniques with the common theme of cultivating mindfulness. Mindfulness has been defined as nonjudgmental, moment-to-moment attention, focused on the present (KabatZinn, 1990). Practices such as the body scan, which is the first clinical practice included in the MBSR program, and sitting meditation are somatically orienting and work to increase one's ability to be attentive and focused. MBSR, as a clinical practice, takes the form of an 8-week course that starts with introductory informational meetings that are followed by eight $2 \frac{1}{2}$ hour long group meetings, and a day long retreat in the sixth week (Kabat-Zinn, 1990). To complete the program, participants are required to practice the techniques they learn in clinic for 45 minutes at home, 6 days a week for the entire duration of the program.

The body scan, also referred to as "sweeping" (Kabat-Zinn, 1982, p.36), involves sitting or lying in a comfortable position while listening to an instructor or a recording that slowly guide attention to different regions of the body. The metaphor commonly used involves a circular furnace slowly scanning a metal bar, and temporarily melting the region in focus, while pushing impurities to the end of the bar. The practice involves using the body as a sort of vehicle to stabilize the mind 
(Dreeben, Mamberg, \& Salmon, 2013). The body scan is one of the fundamental practices that stabilizes attention by shifting focus during insight meditation so it is not lost in thought which can elicit more detailed awareness of emotional, cognitive, and physical experiences (Dreeben et al., 2013). With the body scan specifically, intimate and nonjudgmental knowledge of bodily experience or existence, leads to the acceptance of impermanence, and as a consequence, the dependence on the body as an identification of self is reduced. Dreeben et al. argue that the cognitive shift in dis-identification with the body may be core to the benefits of practicing the body scan in MBSR, as it encourages participants to be flexible and to be able to maintain perspective while perceiving experiences. The body scan as taught in MBSR provides the opportunity to experience the ways in which one has autonomy over judgmental thinking and the flexibility in the quality of attention, which, may contribute to the elimination of suffering (Kabat-Zinn, 1990).

Santarnecchi et al. (2014) measured changes in cortical and subcortical thickness within the brain preceding and anteceding an 8-week training program in MBSR. The study included 23 subjects who were new to meditation. The results from this found significant increases in cortical thickness of the right insula, and significant decreases in self-reported stress and anxiety in the MBSR group compared to the control group (Santarnecchi et al., 2014). According to Uddin et al. (2017), electrical stimulation of the insula has been shown to affect heart rate and blood pressure, indicating a relationship to the autonomic nervous system. Through neuroimaging the insula has also been shown to play a role interception, which is the sense of the physiological condition of the body, demonstrated in subjects who became aware of thirst, heartbeat, and bladder functions (Uddin et al., 2017). The results from the Santarnecchi et al. study involving structural changes within the brains of the MBSR participants have great clinical implications as the findings provide a plausible neurobiological basis for the observed reduction in stress and anxiety that was measured within only 8 weeks.

\section{Methods}

The database PubMed Health, and the search engine Google Scholar were used to identify randomized control trials published since 2015. The keywords used were as follows: ["chronic pain" OR "chronic lower back pain"] AND ["pain management" OR "meditation" OR "alternative treatments"]. The search was limited to studies involving participants with chronic lower back pain meeting the NIH criteria. Combined interventions including mediation along with another method of experimental treatment for chronic pain management were also included. Systematic reviews and meta-analyses were excluded based on the heterogeneity of specific chronic pain conditions. However, meta-analyses were reviewed to search for relevant citations with original data specifically pertaining to chronic lower back pain and meditation. Studies were selected for review if they met all the inclusion criteria as follows: (1) adults with idiopathic chronic lower back pain were involved, (2) were randomized control trials, (3) were group studies, (4) described the group specific outcomes on changes in pain intensity, acceptability, and physical function, (5) used MBSR as an experimental intervention method.

\section{Results}

The single-blind, randomized control study conducted by Morone et al. (2016) assessed the effectiveness of MBSR in the treatment of idiopathic chronic low back pain in older adults. The 8-week study measured changes in function and lower back pain experienced by 282 individuals living in Pittsburgh. The participants were 65 years or older with the average age being 
$74.5,66.3 \%$ were women, predominantly white and all had experiences chronic lower back pain for three months or more, and scored more than 11 points, at baseline, on the Roland and Morris Disability Questionnaire (RMDQ). People who had severely impaired mobility, were experiencing other pain in their body that was more severe than their chronic lower back pain, or people who had moderate to severe depressive states were excluded from the study. The control group consisted of 142 individuals who completed a health education program, and the experimental group included 140 individuals who received an MBSR intervention that included selfmanagement practices such as body scans, walking meditation, and mindful stretching. The primary assessments of the study included changes in mobility and pain which were measured upon completion at week 8- and 6-months post intervention. Participants were also brought in for monthly 60-minute "booster sessions" between the week 8 assessment and the 6-month follow-up.

After 8 weeks Morone et al. (2016) found improvements in function measured by a 1.1 point reduction in the average scores of the intervention group compared to the control group on the RMDQ. At the 6-month follow-up, Morone et al. measured a 0.04 point reduction in the average RMDQ scores of the intervention group compared to the control group. The results measuring improved function in the MBSR group were, however, clinically insignificant $(\mathrm{P}=$ 0.01) when compared to the control group. Morone et al. also found the changes in pain, measured using the Numeric Pain Rating Scale, between the two groups to be clinically insignificant $(\mathrm{P}=$ 0.08 ) at both the 8 week and 6-month follow-up period.

The Luiggi-Hernandez et al. (2017) study was a qualitative analysis of older adults with chronic lower back pain and their experience managing their pain by practicing mindful meditation. The analysis involved qualitative coding of conversations from four different groups, all discussing personal experiences with mindful meditation, in an effort to identify common themes (Luiggi-Hernandez et al., 2017). The study found several common themes involving decreased negative emotions, which including a gained ability to overcome the fear of pain, a shift in pain awareness, and the significance of pain lessened (Luiggi-Hernandez et al., 2017). According to Luiggi-Hernandez et al., based on first hand statements from 25 individuals, ages 65 and older, and who live with chronic lower back pain, mindful meditation may have a positive impact on some older individuals experience with chronic pain.

Cherkin et al. (2016) reported on a randomized controlled trial that assessed the analgesic effects of MBSR and Cognitive-Behavioral Therapy (CBT) as adjunctive methods of treatment for people with chronic lower back pain during a 52-week intervention. The participants initially included 341 patients from an integrated healthcare system in Washington State, who all reported experiencing idiopathic or non-specific lower back pain for at least 3 months (Cherkin et al., 2016). The participants were on average 49.3 years old, $65.7 \%$ were female, $82.5 \%$ were white and $11.1 \%$ were using opioid medication to treat lower back pain (Cherkin et al., 2016). Of the 341 participants 78 of them were unemployed and $37.4 \%$ reported an annual family income less than $\$ 55,000$ USD (Cherkin et al., 2016). Cherkin et al. measured baseline functional limitations using Roland Disability Questionnaire (RDQ), characteristic pain intensity, levels of depression using PHQ-8, and anxiety using GAS-2. The authors also reported that at baseline, only 7.5 out of the 341 participants reported, on a scale from 1 to 10 , that they expected a self-management program would be helpful in alleviating chronic lower back pain. In addition to receiving standard or usual care, 116 participants were trained in MBSR, and 113 participants were treated with CBT (Cherkin et al., 2016). The remaining 112 participants entered the control group, receiving standard care only (Cherkin et al., 2016). Due to loss in follow up, the sample size at 52 weeks, dropped to 264, 
with 88 individuals in each group, which remained large enough to detect significant differences (Cherkin et al., 2016).

Cherkin et al. (2016) found significant between-group improvements in physical function at week 26 between the participants who received MBSR, who scored an average of $60.5 \%$ on the RDQ, and those who received standard care alone, who scored an average of $44.1 \%(P=0.04)$. The trend persisted after 52 weeks, showing significant differences between the MBSR and control group $(P=0.01)$ scores on the RDQ, with the MBSR group scoring 68.6\% and the control group scoring 48.6\% (Cherkin et al., 2016). In comparing pain bothersomeness, Cherkin et al. found significant differences between the MBSR group and the control at weeks $26(\mathrm{P}=0.01)$ and $52(\mathrm{P}$ $=0.02$ ). The authors examined the percentages of participants with significant improvements in pain bothersomeness at week 26 to be $43.6 \%$ and $26.6 \%$ for the MBSR and control groups, respectively. At week 52 the percent of participants with significant improvements in pain bothersomeness were $48.5 \%$ and $31.0 \%$ for the MBSR and control groups, respectively (Cherkin et al., 2016).

Zgierska et al. (2016) conducted a randomized controlled trial that assessed the health benefits of mindful meditation and cognitive behavioral therapy (CBT) during a 26-week intervention period for people with chronic low back pain. The participants in the study were predominantly white women, who were on average 51.8 years old. The majority of the participants received disability-related benefits from the Social Security Administration. Each had been treated with opioid therapy for an average of 7.9 years (Zgierska et al., 2016). Zgierska et al. gathered data on participants' severity of pain using Brief Pain Inventory, their function and disability markers using Oswestry Disability Index, their daily dose of opioid medication, their perceived degree of mindfulness, and their stress levels. Data on the participants' C-reactive protein and cytokine levels and pain sensitivity measures through thermal stimuli was also collected (Zgierska et al., 2016). There were 14 participants included in the MBSR experimental group who, in addition to receiving standard care, participated in eight weekly guided meditation sessions and home practices six days a week, each for 30 minutes. There were also 14 participants in the CBT experimental group and 14 participants in the control group who only received standard care (Zgierska et al., 2016).

Zgierska et al. (2016) found that, on average, the experimental group, including both MBSR and CBT, exhibited $8 \%$ reduction in pain at both week 8 and 26 intervals, compared to the controls, who experienced an $8 \%$ increase in pain by week 8 and a $10 \%$ increase in pain by week $26(P=0.045)$. The results from this study support the practice of meditation as an adjunctive method for chronic pain management (Zgierska et al., 2016). Furthermore, the results from the Zgierska et al. study found significant between-group improvements in physical function and mindfulness, noted as "pain acceptance," between the participants who meditated consistently relative to controls $(P=0.026)$ and those who were inconsistent with their practice relative to controls $(P=0.038)$. These findings show that the benefit of pain reduction may be retained even with infrequent meditative practices.

It may be worth mentioning that the Hilton et al. (2016) meta-analysis neglected to mention the analgesic effects meditation was found to have in the Zgierska et al. (2016) study, and mentioned only the data on morphine equivalence, which was found to be insignificant between the control group and the meditation-CBT groups. The implication of the omission was that the randomized clinical trial was unsuccessful. However, using changes in morphine dosage as a measurement of success may be a poor assessment given the highly individualistic responses to morphine, including physical dependence and tolerance. It may be more useful to compare self- 
assessment of pain while maintaining equipotent doses of morphine for such a study, and therefore reporting on the results found from the "brief pain inventory" (Zgierska et al., 2016) would be most informative.

Kratz, Murphy, Kalpakjian, and Chen (2018) measured the relationship between pain medications, such as opioids, and pain acceptance in 120 adults with chronic pain and spinal cord injuries. Their secondary analysis measured pain severity and distribution using the Brief Pain Inventory, depressive symptoms using the Patient Health Questionnaire-9, in relation to chronic pain acceptance, which was measured using the Chronic Pain Acceptance Questionnaire (Kratz et al., 2018). The study found that greater acceptance of pain was related to lower use of pain medications (Kratz et al., 2018). The authors also found that greater pain distribution was related to greater medication use, and, interestingly, that pain intensity was unrelated to the use of pain medications. The results of this study support the need for therapeutic programs that teach pain acceptance as an approach to chronic pain management, given the lower risks associated with lower pain medication consumption.

A randomized controlled study by Day et al. (2019) compared the feasibility, tolerability, acceptability, and effects of group mindfulness meditation, cognitive therapy, and mindful-based cognitive therapy for 69 patients experiencing chronic low back pain. For each of the treatment groups the results for the post-treatment data showed large improvements in pain interference, pain intensity, physical function and depression ( $\mathrm{P}<0.001$ ) (Day et al., 2019). The follow-up data revealed significantly greater improvements for physical function with in the cognitive therapy treatment group, compared to the mindful meditation group (Day et al., 2019). The results from the study support mindful meditation as an adjunctive method of treatment for chronic lower back pain, as significant improvements in most of the measured categories were found. However, unlike the findings from the Zgierska et al. (2016) study, the results from the Day et al. study suggests that cognitive therapy may be a more effective method of treatment than mindful meditation. Unlike the Zgierska et al. study which compared mindful meditation, and cognitive therapy to standard care alone for a duration of 26 weeks, the Day et al. study lasted only 8 weeks, thus the different findings may be a result in procedural discrepancies.

The Michalsen et al. (2016) randomized control study compared changes in pain intensity, pain-related bothersomeness, physical function, stress levels, quality-of-life and psychological effects of group mindful meditation to a self-care exercise program. The study included 68 patients with chronic lower back pain and lasted a duration of 8 weeks (Michalsen et al., 2016). The study found that both intervention groups experienced a decrease in pain intensity, however there was not a significant between-group difference (Michalsen et al., 2016). However, stress levels were significantly lower in the mindful meditation group compared to the self-care exercise group $(\mathrm{P}=$ 0.011) (Michalsen et al., 2016). The study found no significant between-group effects in painrelated bothersomeness, physical function, quality of life or on the psychological scores. Despite the non-significant statistical findings, Michalsen et al. noted that the mindful meditation group consistently exhibited greater improvements compared to the exercise group intervention.

Ardito et al. (2017) compared salivary cortisol production in an initial group of 17 individuals with chronic lower back pain. Measuring cortisol levels as a stress-related biomarker is a useful technique when comparing treatments for pathologies that are associated with symptoms such as anxiety and depression (McEwen \& Stellar, 1993). Ardito et al. conducted the program at the Department of Pain Management of the Hospital Santa Croce and Carle in Italy. The participants had all experienced at least three months of chronic lower back pain and ranged in ages from 20 to 65 (Ardito et al., 2017). Participants were excluded if they were also receiving 
psychiatric treatments (Ardito et al., 2017). The Ardito et al. study lasted 8 weeks, in which participants completed weekly 2-hour group sessions, at home self-care practices, and during the second to last week they completed a 7-hour group session. The practices included body-scan meditation, sitting and walking meditation as well as yoga exercises (Ardito et al., 2017). As a secondary form of analysis, Ardito et al. measured changes in pain severity, quality of life and depression were measured using the Numeric Rating Scale, the SF-36 Health Status Inventory, and the Beck Depression Inventory, respectively. A total of eight participant completed the entire meditation treatment program and also reported high degree of compliance (Ardito et al., 2017).

Ardito et al. (2017) found that among the eight participants who completed the meditation program, there was no significant difference between baseline and post treatment levels in salivary cortisol measured in the morning $(\mathrm{P}=0.08)$. However, a significant increase from baseline to post treatment levels in salivary cortisol was found in evening measurements $(\mathrm{P}=0.006)$. Despite these findings, all salivary measurements were compared to Cuneo general population, and were consistently lower in the MBSR group than the general population (Ardito et al., 2017). The results may have implications for patients with chronic pain and hypocortisolism, which is a common comorbidity (Ardito et al., 2017). However, decisive analysis of results from the Ardito et al. study are limited due to the extremely small sample size and high dropout rate of individuals included.

The Schmidt et al. (2015) study measured the efficacy of mindfulness-based stress reduction and changes in an EEG pattern that are associated with chronic pain. The study includes 22 patients with chronic lower back who completed an 8-week mindfulness-based stress reduction training (Schmidt et al., 2015). Schmidt et al. found significant changes in health-related life satisfaction $(P=0.01)$, in depression $(P=0.04)$, and affective pain perception $(P=0.04)$ and pain severity $(\mathrm{P}=0.01)$ from baseline measurements to post treatment measurements. However, the EEG analysis revealed no significant changes from the initial time of measurement to the post treatment measurement (Schmidt et al., 2015). The author's findings support mindfulness-based stress reduction as a method of treatment for chronic pain management, however analysis of the results is limited due to the small scope of the study.

\section{Discussion}

Morone et al. (2016) conducted the largest study covered within this literature review, which included 282 participants with chronic lower back pain. The insignificant findings reported in the primary health categories Morone et al. measured, which were mobility and self-assessment of pain, may have related partly to the age criteria for this study, which excluded participants younger than 65 . It is possible that changes in mobility, as a measure of success for the adjunctive treatments being measured, may be a poor primary assessment given other pathologies affecting mobility that are associated with increased age. The time frame of the study, while consistent with the MBSR training, may have also been a limiting factor (Morone et al., 2016). Morone et al. organized the intervention period to last 8 weeks, which was 44 weeks shorter than the study conducted by Cherkin et al. (2016), who found significant results. The two studies had similar sample sizes, but the average age of the participants in the Cherkin et al. study was 25.2 years lower than the average age of participants studied by Morone et al., and Cherkin et al. also organized the intervention period to last 52 weeks.

The qualitative results from Luiggi-Hernandez et al. (2017) were included within this review because their work is complementary to the Morone et al. (2016) study. Although changes in mobility and pain were found to be insignificant based on the results found by Morone et al. the analysis conducted by Luiggi-Hernandez et al. showed that, within a similar demographic (both 
included participants who were 65 or older), individuals may still experience relief from practicing mindful meditation. Commonly expressed themes found by Luiggi-Hernandez et al. within the group of 25 individuals included fewer reports of negative emotions, increased abilities involved in overcoming fear of pain, and noticeable shifts in pain awareness.

The second largest study included in this literature review was conducted by Cherkin et al. (2016) who analyzed the results of 264 participants during and after the conclusion of their 52week long intervention. The authors found significant improvements in the MBSR experimental group in the areas of participant physical limitations and pain bothersomeness compared with the CBT experimental group and the control group. The primary findings from this study support MBSR, as a beneficial adjunctive method of treatment for people with chronic lower back pain (Cherkin et al., 2016). The author's findings are particularly compelling given the large sample size and time frame of the study, as well as the various health sources used to assess the scope and effectiveness of each treatment, through which they were able to show that MBSR may have lasting effects on chronic pain.

Kratz et al. (2018) found that within a study of 120 participants with chronic lower back pain, greater pain acceptance was related to lower use of pain medications. The findings of this study support treatment methods, including mindful meditation pain management, that do not rely on opioid medication, but aim to increase pain acceptance and address depressive symptoms (Kratz et al., 2018). Although the study has a large sample size, it was the only study found that relied on data from a survey, thus the study must be replicated in order to provide more evaluative evidence for the results found from the Kratz et al. cross-sectional survey.

Day et al. (2019) and Michalsen et al. (2016) conducted studies with intervention periods of 8 weeks and had similar sample sizes of 69 and 68, respectively. Both studies found the meditation intervention groups experienced significant decreases in both pain-related bothersomeness and physical function (Day et al., 2019; Michalsen et al., 2016). In comparing a mindful meditation group to an exercise group, Michalsen et al. found no between-group significant differences in pain-related bothersomeness, physical function, or quality of life, or in psychological scores. The findings are not surprising because the study compared two methods of treatment that emphasize self-care. It is likely that the exercise group experienced many of the benefits associated with the practice of mindful meditation, such as self-awareness and, possibly, pain acceptance.

Day et al. (2019) and Zgierska et al. (2016) compared the efficacy of mindful meditation intervention to cognitive therapy as an adjunctive method of chronic pain management. The Day et al. and Zgierska et al. results differ in that Day et al. found that the cognitive therapy group exhibited greater improvements in physical function. These results were not duplicated in the Zgierska et al. study, but the studies' findings were similar in all other measurement outcomes. Zgierska et al. included only 35 study participants, however the duration of the intervention was 26 weeks. The discrepancies in procedural organization and sample size likely contributed to the inconsistent results obtained by Day et al. and Zgierska et al.

Schmidt et al. (2015) and Ardito et al. (2017) had the smallest sample sizes of the studies included with in this review, which were 22 and 8 respectively. Sample sizes equal to or greater than 30 are considered to be sufficiently large and are less likely to have skewed results based on sampling error. Schmidt et al.'s analysis of EEG patterns revealed no significant changes between baseline measurements and measurements taken after week 8 of the MBSR intervention. These findings vary from the results obtained by Santarnecchi et al. (2014). The two studies had nearly identical sample sizes and procedural designs, however Santarnecchi et al. did not use chronic pain 
as an inclusion criterion, and thus was not included within the literature review. Analysis for both the Schmidt et al. and Santarnecchi et al. studies are greatly limited due to the small sample size and narrow intervention periods. While the results obtained by Ardito et al. were even more limited by the small scope of the study, the increased levels of cortisol may be related to the amount of time the participants had experienced chronic pain. The authors imply that the greater the amount of time a person experiences chronic pain, the more likely the HPA axis becomes less responsive to the pain as a stress signal, thus there is a reduction in the serum cortisol levels. The research on HPA axis activity as it pertains to chronic musculoskeletal pain is however somewhat inconclusive (Ardito et al., 2017).

Mindfulness as practiced within the MBSR program, through exercises such as the body scan or sitting meditation, have been found to have numerous physiological effects (Dreeben et al., 2013). For example, Ditto, Eclache, and Goldman (2006) found that the body scan affects parasympathetic activity, which was measured by Respiratory Sinus Arrhythmia (RSA), reflecting heart and breathing rate synchronicity. Skin conductance level (SCL), and peripheral temperature (PT) have also been shown to lower significantly during and post practicing the body scan, implying that body scan, in particular, may lower sympathetic activation (Lush et al. 2009). Significant decreases in overall systolic blood pressure (SBP) and diastolic blood pressure (DBP) have been found in MBSR groups (Nyklíček, Mommersteeg, Van Beugen, Ramakers \& Van Boxtel, 2013). Down regulation of the pro-inflammatory NF- $\kappa B$-related gene expression has frequently been found post-MBSR treatment (Niles, Mehta, Corrigan, Bhasin, \& Denninger, 2014). Gallegos, Lytle, Moynihan, and Talbot (2015) significant decreases in interleukin (IL)-6 levels, another inflammatory biomarker important in the physiological regulation.

Additional treatments and adjunctive methods for chronic pain management are necessary due to the high number of individuals living with chronic pain, the significant economic cost of chronic pain within the U.S., and the risks associated with current treatments. More specifically, the data indicating increased prevalence of people receiving total joint replacement surgeries, a shift to younger ages, and racial discrepancies in (what), shows that further research evaluating alternative methods of chronic pain management is needed to provide adults living with chronic pain with a range of preventative treatment options and to ensure that surgery is utilized as a last result. The observed racial discrepancies, mentioned previously, evoke questions about possible physical overuse and underuse of joints, the balance of information provided to patients of different racial backgrounds, and other confounding variables, such as geography and access, that may influence the disparities found. While many joint replacement surgeries are deemed necessary, especially if there is damage to articular cartilage, it is imperative that surgery, as a form of treatment of chronic joint pain, is not opted for without comprehensive exploration of less invasive and less costly options.

\section{Conclusion}

The literature reviewed indicates that people suffering from chronic lower back pain may benefit from completing the MBSR program while also receiving standard care. Although some results from the literature are consistent with the findings obtained from the University of Massachusetts, Center for Mindfulness Clinic developed by Jon Kabat-Zinn, methodological limitations preclude any definitive conclusions regarding the efficacy of meditation as a method for chronic lower back pain management. The review supports the need for continued research on methods of treatment for chronic pain management that are both valid and reliable, with the goal 
of helping clinicians develop the most effective interventions to help alleviate chronic lower back pain. 


\section{References}

Ardito, R. B., Pirro, P. S., Re, T. S., Bonapace, I., Menardo, V., Bruno, E., \& Gianotti, L. (2017). Mindfulness-based stress reduction program on chronic low-back pain: a study investigating the impact on endocrine, physical, and psychologic functioning. The Journal of Alternative and Complementary Medicine, 23(8), 615-623.

Ballantyne, J. C., \& Mao, J. (2003). Opioid therapy for chronic pain. New England Journal of Medicine, 349(20), 1943-1953.

Banth, S., \& Ardebil, M. D. (2015). Effectiveness of mindfulness meditation on pain and quality of life of patients with chronic low back pain. International Journal of Yoga, 8(2), 128.

Clark, W. C., Yang, J. C., \& Janal, M. N. (1986). Altered pain and visual sensitivity in humans: the effects of acute and chronic stress. Annals of the New York Academy of Sciences, 467(1), 116-129.

Compton, W. M., \& Volkow, N. D. (2006). Major increases in opioid analgesic abuse in the United States: concerns and strategies. Drug and Alcohol Dependence, 81(2), 103-107.

Corder, G., Castro, D. C., Bruchas, M. R., \& Scherrer, G. (2018). Endogenous and exogenous opioids in pain. Annual Review of Neuroscience, 41, 453-473.

Dahlhamer, J., Lucas, J., Zelaya, C., Nahin, R., Mackey, S., DeBar, L., ... Helmick, C. (2018). Prevalence of chronic pain and high-impact chronic pain among adults-United States, 2016. Morbidity and Mortality Weekly Report, 67(36), 1001.

Day, M. A., Ward, L. C., Ehde, D. M., Thorn, B. E., Burns, J., Barnier, A., ... Jensen, M. P. (2019). A pilot randomized controlled trial comparing mindfulness meditation, cognitive therapy, and mindfulness-based cognitive therapy for chronic low back pain. Pain Medicine (Malden, Mass.), 03 January 2019.

Ditto, B., Eclache, M., \& Goldman, N. (2006). Short-term autonomic and cardiovascular effects of mindfulness body scan meditation. Annals of Behavioral Medicine, 32(3), 227-234.

Dreeben, S. J., Mamberg, M. H., \& Salmon, P. (2013). The MBSR body scan in clinical practice. Mindfulness, 4(4), 394-401.

Gamaro, G. D., Xavier, M. H., Denardin, J. D., Pilger, J. A., Ely, D. R., Ferreira, M. B. C., \& Dalmaz, C. (1998). The effects of acute and repeated restraint stress on the nociceptive response in rats. Physiology \& Behavior, 63(4), 693-697.

Gallegos, A. M., Lytle, M. C., Moynihan, J. A., \& Talbot, N. L. (2015). Mindfulness-based stress reduction to enhance psychological functioning and improve inflammatory biomarkers in trauma-exposed women: A pilot study. Psychological Trauma: Theory, Research, Practice, and Policy, 7(6), 525.

Institute of Medicine. (2011). Relieving pain in America: A blueprint for transforming prevention, care, education, and research. The National Academies Collection: Reports funded by National Institutes of Health.

Luiggi-Hernandez, J. G., Woo, J., Hamm, M., Greco, C. M., Weiner, D. K., \& Morone, N. E. (2017). Mindfulness for chronic low back pain: a qualitative analysis. Pain Medicine, 19(11), 2138-2145.

McEwen, B. S., \& Stellar, E. (1993). Stress and the individual: mechanisms leading to disease. Archives of Internal Medicine, 153(18), 2093-2101. 
Michalsen, A., Kunz, N., Jeitler, M., Brunnhuber, S., Meier, L., Lüdtke, R., ... \& Kessler, C. (2016). Effectiveness of focused meditation for patients with chronic low back pain - a randomized controlled clinical trial. Complementary Therapies In Medicine, 26, 79-84.

Morone, N. E., Greco, C. M., Moore, C. G., Rollman, B. L., Lane, B., Morrow, L. A., ... \& Weiner, D. K. (2016). A mind-body program for older adults with chronic low back pain: A randomized clinical trial. JAMA internal medicine, 176(3), 329-337.

NIH MedlinePlus. (11 Feb. 2019). Chronic Pain. Retrieved from medlineplus.gov/chronicpain.html.

Niles, H., Mehta, D. H., Corrigan, A. A., Bhasin, M. K., \& Denninger, J. W. (2014). Functional genomics in the study of mind-body therapies. Ochsner Journal, 14(4), 681-695.

Nyklíček, I., Mommersteeg, P., Van Beugen, S., Ramakers, C., \& Van Boxtel, G. J. (2013). Mindfulness-based stress reduction and physiological activity during acute stress: A randomized controlled trial. Health Psychology, 32(10), 1110.

Hilton, L., Hempel, S., Ewing, B. A., Apaydin, E., Xenakis, L., Newberry, S., ... \& Maglione, M. A. (2016). Mindfulness meditation for chronic pain: systematic review and meta-analysis. Annals of Behavioral Medicine, 51(2), 199-213.

Hoots, B. E., Xu, L., Kariisa, M., Wilson, N. O., Rudd, R. A., Scholl, L., ... \& Seth, P. (2018). 2018 Annual surveillance report of drug-related risks and outcomes--United States.

Kabat-Zinn, J. (1990). Full Catastrophe Living. New York: Bantam Dell.

Kabat-Zinn, J. (2003). Mindfulness-based interventions in context: Past, present, and future. Clinical Psychology: Science and Practice, 10(2), 144-156.

Kabat-Zinn, J., Lipworth, L., \& Burney, R. (1985). The clinical use of mindfulness meditation for the self-regulation of chronic pain. Journal of Behavioral Medicine, 8(2), 163-190.

Kabat-Zinn, J. (1982). An outpatient program in behavioral medicine for chronic pain patients based on the practice of mindfulness meditation: theoretical considerations and preliminary results. General Hospital Psychiatry, 4(1), 3347.

Kratz, A. L., F Murphy, J., Kalpakjian, C. Z., \& Chen, P. (2018). Medicate or meditate? greater pain acceptance is related to lower pain medication use in persons with chronic pain and spinal cord injury. The Clinical Journal of Pain, 34(4), 357-365.

Krebs, E. E., Gravely, A., Nugent, S., Jensen, A. C., DeRonne, B., Goldsmith, E. S., ... \& Noorbaloochi, S. (2018). Effect of opioid vs. non opioid medications on pain-related function in patients with chronic back pain or hip or knee osteoarthritis pain: the SPACE randomized clinical trial. JAMA, 319(9), 872-882.

Kremers, H. M., Larson, D. R., Crowson, C. S., Kremers, W. K., Washington, R. E., Steiner, C. A., ... \& Berry, D. J. (2015). Prevalence of total hip and knee replacement in the United States. The Journal of Bone and Joint Surgery. American volume, 97(17), 1386.

Lush, E., Salmon, P., Floyd, A., Studts, J. L., Weissbecker, I., \& Sephton, S. E. (2009). Mindfulness meditation for symptom reduction in fibromyalgia: Psychophysiological correlates. Journal of Clinical Psychology in Medical Settings, 16(2), 200.

Schappert SM, Burt CW. Ambulatory care visits to physician offices, hospital outpatient departments, and emergency departments: United States, 2001-02. Vital Health Stat 13 2006;13: 5,18. PubMed 
Santarnecchi, E., D’Arista, S., Egiziano, E., Gardi, C., Petrosino, R., Vatti, G., ... Rossi, A. (2014). Interaction between neuroanatomical and psychological changes after mindfulness-based training. PloS one, 9(10), e108359.

Schmidt, S., Gmeiner, S., Schultz, C., Löwer, M., Kuhn, K., Naranjo, J. R., ... Hinterberger, T. (2015). Mindfulnessbased Stress Reduction (MBSR) as treatment for chronic back pain-an observational study with assessment of thalamocortical dysrhythmia. Complementary Medicine Research, 22(5), 298-303.

Seth, P., Scholl, L., Rudd, R. A., \& Bacon, S. (2018). Overdose deaths involving opioids, cocaine, and psychostimulants-United States, 2015-2016. Morbidity and Mortality Weekly Report, 67(12), 349.

Tomek, I. M., Goodman, D. C., Esty, A. R., Bell, J. E., \& Fisher, E. S. (2010). Trends and regional variation in hip, knee and shoulder replacement. The Dartmouth Institute for Health Policy and Clinical Practice.

Uddin, L. Q., Nomi, J. S., Hébert-Seropian, B., Ghaziri, J., \& Boucher, O. (2017). Structure and function of the human insula. Journal of Clinical Neurophysiology, 34(4), 300-306.

Volkow, N. D., \& McLellan, A. T. (2016). Opioid abuse in chronic pain-misconceptions and mitigation strategies. New England Journal of Medicine, 374(13), 1253-1263.

Wilder-Smith, O. H., \& Arendt-Nielsen, L. (2006). Postoperative hyperalgesia its clinical importance and relevance. Anesthesiology: The Journal of the American Society of Anesthesiologists, 104(3), 601-607.

Young, S. (1994). Purpose and method of vipassana meditation. The Humanistic Psychologist, 22(1), 53-61. doi:http://dx.doi.org.proxy.lib.pdx.edu/10.1080/08873267.1994.9976936

Zgierska, A. E., Burzinski, C. A., Cox, J., Kloke, J., Stegner, A., Cook, D. B., ... \& Bačkonja, M. (2016).

Mindfulness meditation and cognitive behavioral therapy intervention reduces pain severity and sensitivity in opioid-treated chronic low back pain: pilot findings from a randomized controlled trial. Pain Medicine, 17(10), $1865-1881$. 